\title{
ARTICLE
}

\section{Numerical simulation of BL06 neutron beamline for "VIN ROSE" at J-PARC/MLF}

\author{
Tatsuro Oda $^{\mathrm{a}^{*}}$, Masahiro Hino ${ }^{\mathrm{b}}$, Masaaki Kitaguchi ${ }^{\mathrm{b}}$, Yuji Kawabata ${ }^{\mathrm{b}}$, Norifumi L. Yamada ${ }^{\mathrm{c}}$ and Hideki Seto $^{\mathrm{c}}$ \\ ${ }^{a}$ Department of Nuclear Engineering, Kyoto University, Nishikyo-ku, Kyoto 615-8530, Japan; ${ }^{b}$ Research Reactor Institute, Kyoto \\ University (KURRI), Kumatori, Sennan-gun, Osaka 590-0494, Japan; ${ }^{~}$ Neutron Science Laboratory, High Energy Accelerator \\ Research Organization (KEK), Tokai, Naka-gun, Ibaraki 319-1106, Japan
}

\begin{abstract}
A new beam line for neutron resonance spin echo spectrometers is under construction at BL06 in the Materials and Life Science Facility (MLF) of the Japan Proton Accelerator Research Complex (J-PARC). To design the radiation shielding in the setup for commissioning experiments we have performed the neutron beam transport calculation and dose evaluation via Monte Carlo method in the three-dimensional geometry. The radiation dose rates were kept below about $6 \mu \mathrm{Sv} / \mathrm{h}$ outside of the thickness of $30 \mathrm{~cm}$ concrete shield surrounding the experimental area. We propose the conceptual design of radiation shielding of the beam line and simulation result playing an important role in determining engineering design and starting the construction.
\end{abstract}

\section{Keywords: J-PARC; shielding design; neutronics; neutron guide; neutron supermirror; neutron resonance spin echo; $M I E Z E$}

\section{Introduction}

Kyoto University and KEK started to construct a new beam line for neutron spin echo (NSE) spectrometers at the BL06 in the Materials and Life Science Facility (MLF) of the Japan Proton Accelerator Research Complex (J-PARC). NSE method proposed by F. Mezei [1] is a powerful tool to investigate slow dynamics of condensed matter. It directly measures intermediate scattering function $S(q, t)$ with very high neutron energy resolution. In order to cover wide range of energy and momentum transfer with various sample environments, two types of NSE spectrometers will be installed in the BL06: NRSE (Neutron Resonance Spin Echo) [2, 3] and MIEZE (Modulated Intensity by Zero Effort) [4]. NRSE is suitable to study slow dynamics of soft condensed matter with high energy resolution. MIEZE has a big advantage of flexible sample environments with potential to open new fields of study. Thus we named them "VIN ROSE" (Village of Neutron Resonance Spin Echo Spectrometers).

For the construction and start of experiments, it is necessary to estimate the radiation shielding performance of the beam line to fulfill the legal requirements and the rules of MLF concerning the prevention of radiation hazards. The limit value of dose rate is $12.5 \mu \mathrm{Sv} / \mathrm{h}$ in areas where experimenters can enter during beam operation [5]. Because the

*Corresponding author. Email: t_oda@ nucleng.kyoto-u.ac.jp instruments of VIN ROSE are comprised of considerable number of neutron optical devices such as beam slit, polarizing and analyzing mirrors, spin flippers, focusing mirrors and guide field coils, it is difficult to set all components in calculation and we cannot fix them before tuning using neutron beam. In this study, we propose the shielding design for the commissioning phase before the fully-equipped common use of VIN ROSE, and calculate radiation dose rate and neutron beam property to ensure that the dose level bellows the regulation limit in commissioning experiments. After the construction and commissioning, vacuum flight path and focusing mirror for beam correction in NRSE [6] will be installed and they should suppress dose rate due to neutrons scattered in air. Numerical simulations are done by using PHITS (Particle and Heavy Ion Transport code System), a Monte Carlo simulation code [7, 8].

\section{Concept of beam line and shielding design}

Figure 1 shows the schematic view of VIN ROSE. The letter $z$ indicates the distance from the source, and the center of the source duct was at the position of $\mathrm{x}=0$, $y=0$. The spallation neutron source of MLF produces neutrons in very wide energy region up to $1 \mathrm{GeV}$. Fast neutrons cause of high dose rate and call for space-occupying massive shield. In the aim of stopping such fast neutrons and gamma rays from the source within iron pre-shield positioned at $\mathrm{z}=7.2-12 \mathrm{~m}$, and 


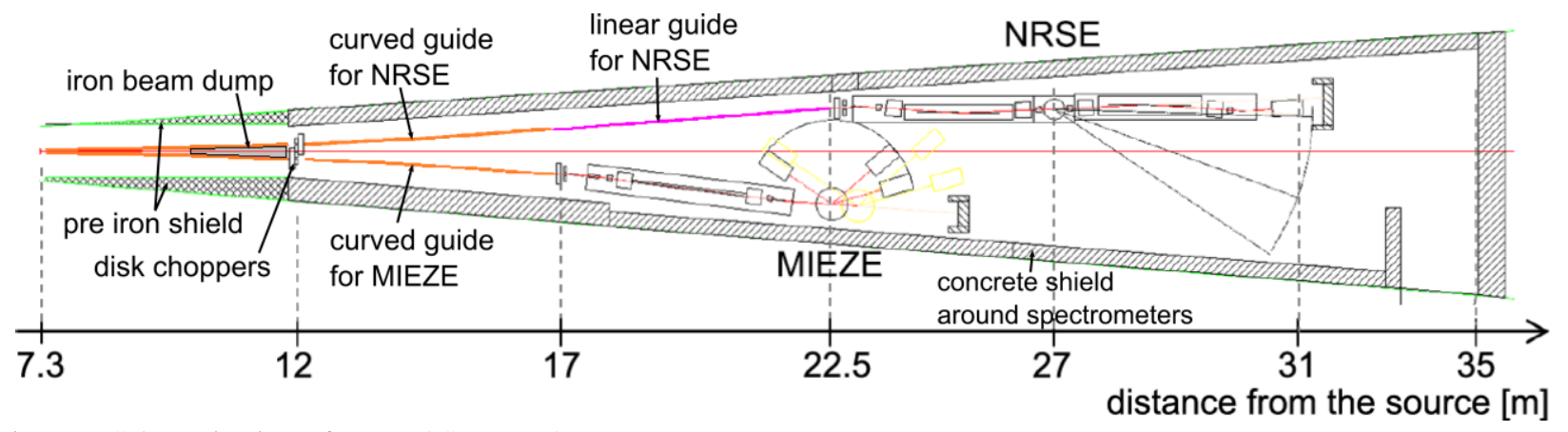

Figure 1. Schematic view of VIN ROSE at BL06

transportation of optimized slow neutron beam, we set two curved neutron guide $(\mathrm{z}=7.3-17.1 \mathrm{~m}$, the curvature radii are $140 \mathrm{~m}$ ) and an iron beam dump between the branch. For high resolution measurement, it is important to transport neutrons with long wavelength since the energy resolution of NSE method is proportional to the third power of neutron wavelength. In the NRSE guide, a linear guide extends from $\mathrm{z}=17.3$ to $22.7 \mathrm{~m}$. NRSE guide has elliptical focusing shapes in vertical direction $(\mathrm{z}=7.3-22.7 \mathrm{~m})$ and in horizontal direction $(\mathrm{z}=$ 17.3-22.7 m). The guide tubes are made of supermirrors sputtered on the base of silicon wafer and they are covered by steel shields to stop gamma radiations from the neutron capture reaction. The guide tubes of neutron suppermirror of $m=2$ with a cross-section of $93 \mathrm{~cm} \times 93$ $\mathrm{cm}$ was inserted into the shutter and the biological shield $(\mathrm{z}=2.3-4.3,4.3-7.2 \mathrm{~m})$. Here, the number of $m$ indicates the unit of a critical reflection momentum of natural $\mathrm{Ni}$. The performance of suppermirros of NRSE guide and MIEZE guide are $m=2.5$ and $m=3$, respectively. The disk choppers to select wavelength band will be set at the position of $\mathrm{z}=12.3 \mathrm{~m}$ and longer wavelength will be cut by filtering mirror.

Figure 2 shows the present shielding design and the geometry in the calculation of PHITS. Beam stoppers made of $\mathrm{B}_{4} \mathrm{C}$-containing rubber and lead with thickness of $5 \mathrm{~mm}$ and a few $\mathrm{cm}$, respectively were set at the position $\mathrm{z}=25 \mathrm{~m}$ and $\mathrm{z}=31 \mathrm{~m}$ for MIEZE and NRSE, respectively, and this location is the same as the fully-equipped setup in future. Polarizing mirrors and beam slits were set at the guide exit of NRSE and MIEZE, and the housing consisting of B4C rubber and lead was set to stop scattered neutrons and gamma rays from mirror or slit as shown in Figure 2 (c).

\section{Geometry and conditions of numerical simulation}

The proton beam power was assumed to be $1 \mathrm{MW}$ and the neutron source spectrum was set as the data given by the Web site of MLF [9]. The Monte Carlo simulations have done by using PHITS 2.30 and nuclear data was JENDL-4.0. The dose rate was calculated from neutron and photon flux multiplied by conversion factors embedded in PHITS [10, 11].

In this calculation the thickness of concrete from $\mathrm{z}=$ 16 to $\mathrm{z}=35 \mathrm{~m}$ (except the backmost concrete wall) around experimental area was changed 10, 20 and $30 \mathrm{~cm}$
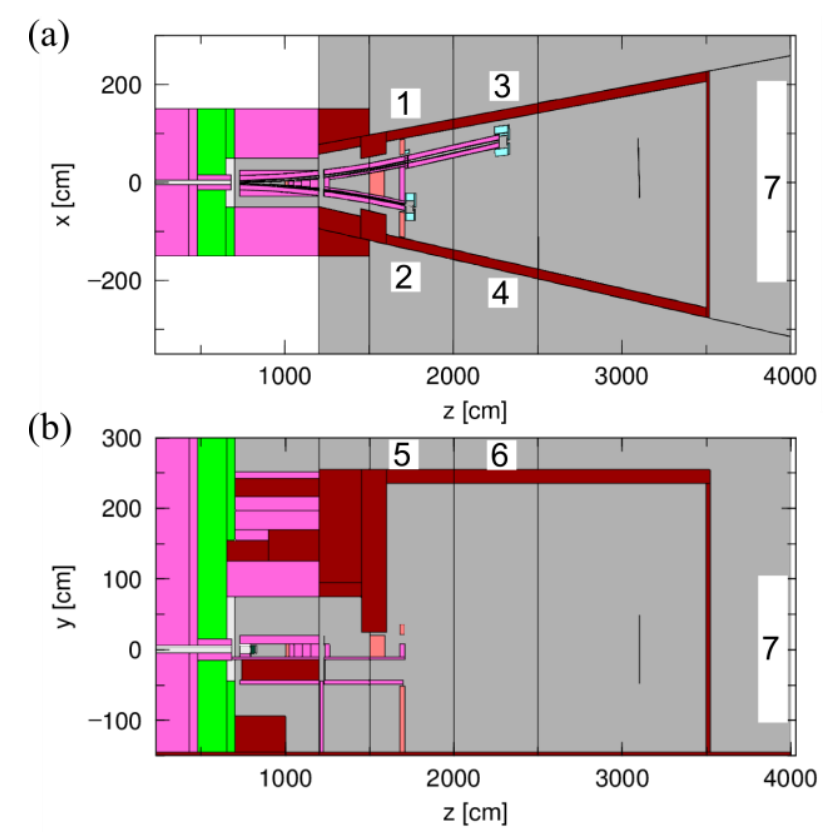

(c)
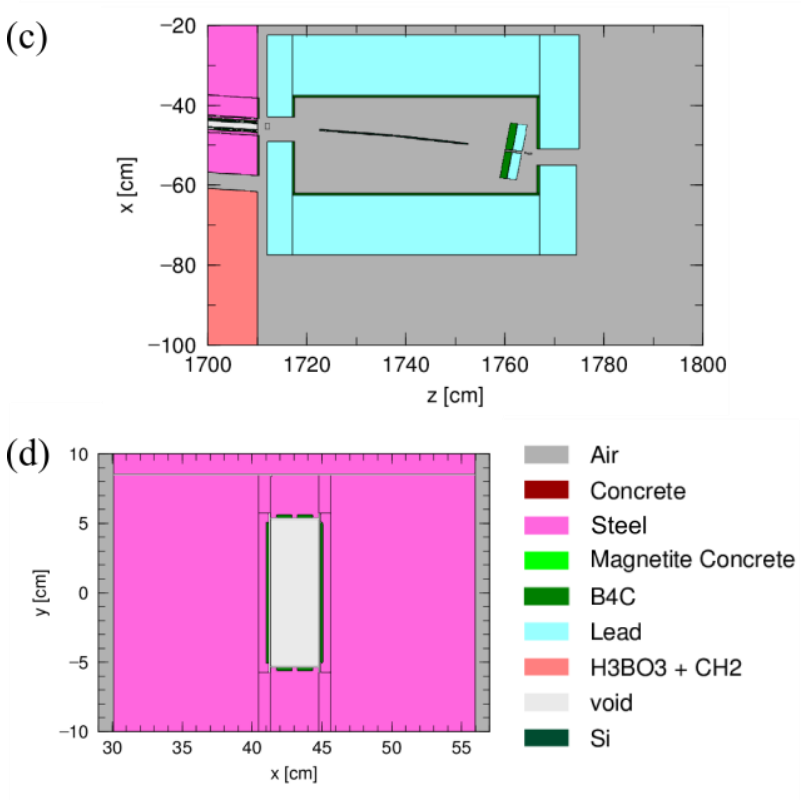

Figure 2. Present design of shielding of BL06 and the geometry of PHITS calculation in (a) horizontal (xz plane) view and (b) vertical (yz plane) view. (c) Housing shield for the polarizing mirror and beam slit for the MIEZE instrument. (d) Cross-section in downstream of NRSE guide. The numbered positions are selected to indicate the exemplary value of calculated dose rate. 
to optimize the shield volume. The thickness of the backmost concrete wall was changed 40 and $50 \mathrm{~cm}$, which is thicker than side shields so as to suppress dose value at the boundary of the MLF experimental hall.

\section{Simulation results}

The estimated neutron spectrums at variety of positions of the neutron guides are shown in Figure 3. Very few fast neutrons appear each guide exit and only slow neutrons $(<100 \mathrm{meV})$ are obtained due to the selective function of the curved guide. Neutron intensity was expected to be about $2 \times 10^{8} \mathrm{n} / \mathrm{cm}^{2} / \mathrm{s} / \AA$ at each guide exit in the $1 \mathrm{MW}$ operation, and peak wavelength are $3.5 \AA$ and $5.2 \AA$ for MIEZE and NRSE respectively.

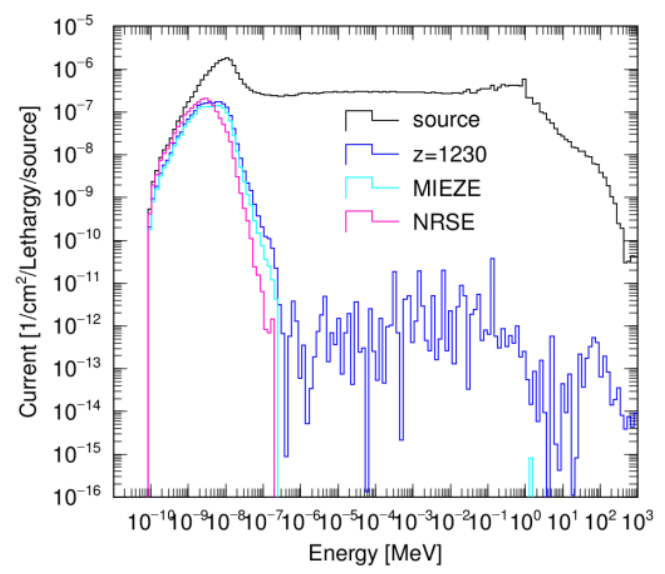

Figure 3. The neutron spectrums at the source, the position of $\mathrm{z}=12.3 \mathrm{~m}$, guide exits of $\operatorname{MIEZE}(\mathrm{z}=17.3 \mathrm{~m})$ and NRSE $(\mathrm{z}=22.7 \mathrm{~m})$.
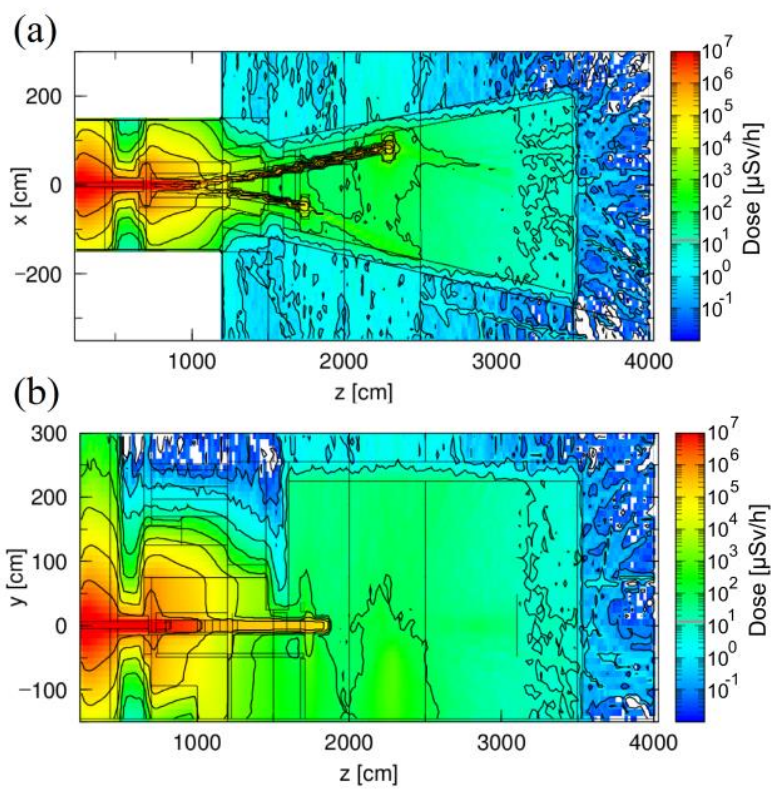

Figure 4. Total dose rate map (neutron + photon) in (a) horizontal (xz plane) view and (b) vertical (yz plane) view in the present shielding design. The gray line in color bar indicates regulatory limit dose value of $12.5 \mu \mathrm{Sv} / \mathrm{h}$.
Figure 4 illustrates the two-dimensional distribution of radiation dose rate converted from neutron and photon flux in the setup for commissioning experiment. The dose rate value at the positions indicated in Figure 2 (a), (b) are written in Table 1. In terms of the regulatory limit dose value of $12.5 \mu \mathrm{Sv} / \mathrm{h}$, the side concrete thickness of $10 \mathrm{~cm}$ was not acceptable and side concrete shield with thickness of more than $20 \mathrm{~cm}$ suppressed dose rate below $10 \mu \mathrm{Sv} / \mathrm{h}$. From these results, the thickness of $30 \mathrm{~cm}$ is considered appropriate for concrete shielding around the spectrometers with some safety margin.

Table 1. Calculated dose rate in $[\mu \mathrm{Sv} / \mathrm{h}]$ at the positions of evaluation (shown in Figure 2.) for variety of thickness of concrete shield surrounding experimental area of BL06.

\begin{tabular}{cccc}
\hline $\begin{array}{c}\text { Position of } \\
\text { evaluation }\end{array}$ & $\begin{array}{c}\text { Concrete } \\
10 \mathrm{~cm}\end{array}$ & $\begin{array}{c}\text { Concrete } \\
20 \mathrm{~cm}\end{array}$ & $\begin{array}{c}\text { Concrete } \\
30 \mathrm{~cm}\end{array}$ \\
\hline No. 1 & 4.9 & 2.3 & 1.4 \\
No. 2 & 6.6 & 3.5 & 2.0 \\
No. 3 & 15.6 & 10.3 & 5.8 \\
No. 4 & 12.5 & 7.9 & 4.1 \\
No. 5 & 7.1 & 6.4 & 3.9 \\
No. 6 & 24.2 & 8.1 & 2.9 \\
\hline
\end{tabular}

In the both case of back concrete wall $(\mathrm{z}=35 \mathrm{~m})$ with thickness of $40 \mathrm{~cm}$ and $50 \mathrm{~cm}$, the dose rata at the position No.7 was $0.06 \mu \mathrm{Sv} / \mathrm{h}$, and the thicknesses of side and ceiling concrete were set at $30 \mathrm{~cm}$. This value was calculated by averaging over $10 \mathrm{~cm} \times 10 \mathrm{~cm}$ mesh points in the $\mathrm{xz}$ plane in $400 \times 100 \times 100 \mathrm{~cm}^{3}$ area in the $x y z$ space $(x=-200-200, y=-50-50, z=3800-3900 \mathrm{~cm})$, and the variance of dose rate of these mesh points was about $\pm 0.1 \mu \mathrm{Sv} / \mathrm{h}$. This large variance came from photon dose, so in order to suppress it, additional steel shielding may be needed.

\section{Summary}

We have performed Monte Carlo simulations to design the radiation shielding of a new beam line for neutron resonance spin echo spectrometers at BL06 in J-PARC/MLF. In the present shielding design for the commissioning phase, the dose rate was estimated about $10 \mu \mathrm{Sv} / \mathrm{h}$ at side of the experimental area of BL06. At near the boundary of MLF experimental hall, the dose rate is in the order of $0.1 \mu \mathrm{Sv} / \mathrm{h}$. Engineering design and construction of the shielding will be done based on the conceptual design and findings proposed in this study.

\section{Acknowledgement}

This study was supported by Grant-in-Aid for Scientific Research Grant No. 23360428 of JSPS. The construction of the BL06 beam line for VIN ROSE was supported by the Neutron Scattering Program Advisory Committee of IMSS, KEK (Proposal No. 2009S07). 


\section{References}

[1] F. Mezei, Neutron spin echo: A new concept in polarized thermal neutron techniques, Z. Phys. 255 (1972), pp.146-160.

[2] R. Golub and R. Gähler, A neutron resonance spin echo spectrometer for quasi-elastic and inelastic scattering, Phys. Lett. A 123 (1987), pp.43-48.

[3] R. Gähler and R. Golub, A high resolution neutron spectrometer for quasielastic scattering on the basis of spin-echo and magnetic resonance, Z. Phys. B 65 (1987), pp. 269-273.

[4] R. Gähler, R. Golub and T. Keller, Neutron resonance spin echo-a new tool for high resolution spectroscopy, Phys. B 180 (1992), pp.899-902.

[5] M. Harada, K. Oikawa, Y. Kasugai and F. Maekawa, Shielding design of a neutron beam line "NOBORU" at JSNS/J-PARC, Prog. Nucl. Sci,. Technol. 1 (2011), pp.94-97.

[6] M. Bleuel, F. Demmel, R. Gähler, R. Golub, K. Habicht, T. Keller, S. Klimko, I. Köper, S. Longeville and S. Prokudaylo, Neutron spin echo spectroscopy, Lecture Notes in Physics, vol.601, Springer, Berlin, (2003), p.176.
[7] K. Niita, N. Matsuda, Y. Iwamoto, H. Iwase, T. Sato, H. Nakashima, Y. Sakamoto and L. Sihver, PHITS: Particle and Heavy Ion Transport code System, Version 2.23, JAEA-Data/Code 2010-022 (2010).

[8] K. Mishima, T. Ino, K. Sakai, T. Shinohara, K. Hirota, K. Ikeda, H. Sato, Y. Otake, H. Ohmori, S. Muto, N. Higashi, T. Morishima, M. Kitaguchi, M. Hino, H. Funahashi, T. Shima, J. Suzuki, K. Nita, K. Taketani, Y. Seki and H. M. Shimizu, Design of neutron beamline for fundamental physics at J-PARC BL05, Nucl. Instr. Meth. A 600 (2009), pp. 342-345.

[9] Web site of J-PARC/MLF, http://j-parc.jp/researcher/MatLife/ja/instrumentati on/

[10] Y. Sakamoto and Y. Yamaguchi, Dose conversion coefficients in the shielding design calculation for high energy proton accelerator facilities, JAERI-Tech 2001-042, (2001).

[11] ICRP, Conversion coefficients for use in radiological protection against external radiation, ICRP Publication 74, (1996). 